\title{
Coexisting Respiratory Comorbidities Increase Mortality in Patients with Asthma: A National Cohort Study
}

\author{
Yoomi Yeo ${ }^{1}$, Dong Won Park ${ }^{1}$, Jiin Ryu ${ }^{1}$, Sung Jun Chung ${ }^{1}$, Tai Sun Park ${ }^{1}$, Hyun Lee ${ }^{1}$, \\ Sang-Heon Kim ${ }^{1}$, Jang Won Sohn ${ }^{1}$, Ho-Joo Yoon ${ }^{1}$, Tae-Hyung Kim ${ }^{1}$, and Ji-yong MOON ${ }^{1}$ \\ ${ }^{1}$ Hanyang University
}

October 26, 2020

\begin{abstract}
Background: Asthmatic patients are generally considered to have an increased risk of mortality compared with subjects without asthma. However, this issue has not been fully evaluated using nationally representative data. In addition, it is unclear whether respiratory comorbidities other than chronic obstructive pulmonary disease (COPD) are associated with increased mortality in asthmatic patients compared with subjects without asthma. Methods: Using a nationally representative sample database, we performed a retrospective cohort study of patients with asthma and age- sex-matched control cohort. We estimated hazard ratio (HR) and 95\% confidence interval (CI) for mortality. We also stratified the asthma cohort based on respiratory comorbidities. Results: During a median 8.9-year follow-up, the overall mortality rate was higher in the asthma cohort than in the control cohort $(\mathrm{p}<0.001)$. The HR for mortality in the asthma cohort compared with the control cohort was $1.13(95 \%$ CI $=1.07-$ 1.19). The effects of asthma on mortality were more evident in males, patients under medical aid, and subjects with COPD. Respiratory comorbidities were significantly associated with increased risk of mortality in asthmatic patients compared with controls $(\mathrm{COPD}$, adjusted $\mathrm{HR}$, aHR $=1.49,95 \% \mathrm{CI}=1.39-1.59$; bronchiectasis, aHR $=1.43,95 \% \mathrm{CI}=1.18-1.73$; lung cancer, $\mathrm{aHR}=4.11,95 \% \mathrm{CI}=2.59-6.52 ;$ pneumonia, $\mathrm{aHR}=1.59,95 \% \mathrm{CI}=1.46-1.74)$. Conclusion: Patients with asthma had a higher mortality rate compared with subjects without asthma. Coexisting pulmonary comorbidities were the primary cause of higher mortality in patients with asthma
\end{abstract}

\section{Coexisting Respiratory Comorbidities Increase Mortality in Patients with Asthma: A National Cohort Study}

Short title: Increased mortality in asthma with pulmonary comorbidity

Yoomi Yeo ${ }^{1 *}$, Hyun Lee ${ }^{*}$, Jiin Ryu ${ }^{2 *}$, Sung Jun Chung ${ }^{1}$, Tai Sun Park ${ }^{1}$, Dong Won Park ${ }^{1}$, Sang-Heon $\mathrm{Kim}^{1}$, Tae Hyung Kim ${ }^{1}$, Jang Won Sohn ${ }^{1}$, Ho Joo Yoon ${ }^{1}$, Ji-Yong Moon ${ }^{1}$

${ }^{1}$ Department of Internal Medicine, Hanyang University College of Medicine, Seoul, Korea

${ }^{2}$ Biostatistical Consulting and Research Lab, Medical Research Collaborating Center,

Hanyang University, Seoul, Korea

${ }^{*}$ Yeo Y., Lee H., and Ryu J. contributed to this work equally.

Word count of the manuscript text: 2,539

Number of tables: 2

Number of figures: 3

ACKNOWLEDGEMENTS 
This work was supported by a National Research Foundation of Korea (NRF) grant funded by the Korean government (MSIT) (No. 2020R1F1A1070468 to H. Lee)

\section{Funding information}

This work was supported by a National Research Foundation of Korea (NRF) grant funded by the Korean government (MSIT; No. 2020R1F1A1070468 to H. Lee).

\section{Corresponding author:}

Ji-Yong Moon, M.D., Ph.D.

Division of Pulmonology, Allergy, and Critical Care Medicine, Department of Internal Medicine

Hanyang University Guri Hospital, Hanyang University College of Medicine

153, Gyeongchun-ro, Guri-si, Gyeonggi-do, Korea (11923)

Mobile: (82) 10-2018-1018; TEL: (82) 31-560-2244; FAX: (82) 31-557-2758

Email: respiry@gmail.com

\section{ABSTRACT}

Background: Asthmatic patients are generally considered to have an increased risk of mortality compared with subjects without asthma. However, this issue has not been fully evaluated using nationally representative data. In addition, it is unclear whether respiratory comorbidities other than chronic obstructive pulmonary disease (COPD) are associated with increased mortality in asthmatic patients compared with subjects without asthma.

Methods: Using a nationally representative sample database, we performed a retrospective cohort study of patients with asthma and age- sex-matched control cohort. We estimated hazard ratio (HR) and 95\% confidence interval (CI) for mortality. We also stratified the asthma cohort based on respiratory comorbidities.

Results: During a median 8.9-year follow-up, the overall mortality rate was higher in the asthma cohort than in the control cohort $(\mathrm{p}<0.001)$. The HR for mortality in the asthma cohort compared with the control cohort was $1.13(95 \% \mathrm{CI}=1.07-1.19)$. The effects of asthma on mortality were more evident in males, patients under medical aid, and subjects with COPD. Respiratory comorbidities were significantly associated with increased risk of mortality in asthmatic patients compared with controls (COPD, adjusted $\mathrm{HR}, \mathrm{aHR}=1.49,95 \% \mathrm{CI}=1.39-1.59 ;$ bronchiectasis, aHR $=1.43,95 \% \mathrm{CI}=1.18-1.73$; lung cancer, aHR $=4.11,95 \% \mathrm{CI}=2.59-6.52$; pneumonia, $\mathrm{aHR}=1.59,95 \% \mathrm{CI}=1.46-1.74)$.

Conclusion: Patients with asthma had a higher mortality rate compared with subjects without asthma. Coexisting pulmonary comorbidities were the primary cause of higher mortality in patients with asthma

Keywords: asthma, mortality, respiratory comorbidities

\section{Abbreviations}

aHR adjusted hazard ratio

CI confidence interval

COPD chronic obstructive pulmonary disease

ICD-10 10th revision of the International Statistical Classification of Diseases and

Related Health Problems

NHIS-NSC National Health Insurance System-National Sample Cohort

NTM non-tuberculous mycobacteria 


\section{INTRODUCTION}

Asthma affects $5-10 \%$ of the population and contributes to approximately 0.4 million deaths annually worldwide $^{1}$. Asthma causes a high global burden of death and disability and is in the top 20 causes of years of life lived with disability. Although the mortality in asthmatic patients has decreased, asthma still has a sizable health-related burden of life $\mathrm{e}^{2,3}$.

Asthmatic patients are generally considered to have higher mortality than subjects without asthma ${ }^{4-6}$. In a large population-based matched cohort study, the mortality in asthmatics was higher than in controls ${ }^{4}$. Another study reported similar results using a provincial health database ${ }^{6}$. However, in most countries, it remains inconclusive which factors cause higher mortality in patients with asthma compared with subjects without asthma because of the lack of evaluation of this issue using a nationally representative database ${ }^{4,6}$.

Respiratory conditions including chronic obstructive pulmonary disease (COPD), bronchiectasis, lung cancer, pneumonia, and non-tuberculous mycobacteria (NTM) infection are important comorbidities that affect the natural course of asthma. Coexisting COPD is associated with poor asthma control and increased healthcare use and is an important risk factor for mortality in patients with asthma ${ }^{4,6}$. However, except for COPD, the association of these comorbidities and mortality in patients with asthma has not been well elucidated, although the comorbid conditions have potential deteriorating effects of asthma on mortality. For example, bronchiectasis is associated with the severity of asthma ${ }^{7}$, and asthmatic patients are at increased risk of serious diseases, such as lung cancer ${ }^{8}$ and pulmonary infectious conditions (e.g., pneumonia and NTM infection $)^{9,10}$, which can result in increased mortality in patients with asthma.

Thus, this study aimed to compare the mortality rate in patients with and without asthma using a representative nationwide database. We also evaluated the effects of asthma-related respiratory comorbidities on mortality in patients with asthma compared with subjects without asthma.

\section{MATERIALS AND METHODS}

\subsection{National Health Insurance System (NHIS)-National Sample Cohort (NSC)}

In South Korea, almost all Korean citizens are enrolled in the National Health Insurance System (NHIS). The NHIS collects health data from nearly 50 million of the insured subjects, including admission and outpatient visit records, diagnoses, drug prescriptions, national health examination data, death, and causes of death. The NHIS provides all the above-mentioned information for research purposes. For the present study, we used data from the NHIS-National Sample Cohort (NSC), a specialized data set provided by NHIS that includes a stratified random sample for the total eligible Korean population (approximately 1 million people $)^{11}$.

\subsection{Definitions}

Adult asthma was defined based on the following criteria: (1) 18 years of age or older, (2) at least two claims under the 10th revision of the International Statistical Classification of Diseases and Related Health Problems (ICD-10) codes J45-46, and (3) at least one claim for prescription of asthma-related drugs including inhaled or systemic corticosteroids, bronchodilators, leukotriene receptor antagonists, or xanthine derivatives ${ }^{12,13}$. The index event for incident asthma was defined as the first prescription of asthma-related medication with ICD codes J45-46 as a major or minor diagnosis between January 1, 2005, and December 31, 2006, with no diagnosis code more than three years before the first index event after the sensitivity test. The baseline period was defined as 12 months before the index date. The follow-up period was from the index date to the date of death or December 31, 2015.

\subsection{Study population}

As shown in Supplemental Figure 1, 738,254 patients 18 years of age or older were identified from January 1, 2005 to December 31, 2006. After excluding 16,995 subjects with malignancy during the washout period, 697,281 patients were selected. Among these, 80,356 patients had at least one claim for ICD-10 codes for asthma (J56-46). 
Among the 80,356 patients with asthma, we excluded patients who had asthma during the three-year washout period $(\mathrm{n}=15,069)$, patients who had only one claim under ICD-10 codes for asthma $(\mathrm{n}=28,333)$, patients who did not receive asthma-related medication $(\mathrm{n}=16,592)$, and patients who died during the baseline period $(\mathrm{n}=55)$. Finally, a total of 19,319 patients with incident asthma was identified (asthma cohort). Next, we performed 1:4 age and sex matching for each asthma patient from cohort without asthma (control cohort). Patients were followed-up until the date of death or December 31, 2015.

This study was approved by the Ethics and Review Board of Hanyang University Guri Hospital (IRB No. 2019-11-018). The requirement of informed consent from the participants was waived because the NHIS database was constructed after anonymization.

\subsection{Comorbidities}

Baseline comorbidities were defined as comorbidities with at least one claim under ICD-10 codes using a major or minor diagnosis during the baseline period as follows: respiratory diseases (J00-J99) including COPD (J43 or J44 except for J43.0), bronchiectasis (J47), NTM infection (A3), and pneumonia (J12-J18); cardiovascular diseases (I00-I99) including hypertension (I10-I15), ischemic heart diseases (I20-I25) including angina (I20) and myocardial infarction (I21), and congestive heart failure (I50); cerebrovascular diseases (I60I69); endocrine diseases (E00-E90) including diabetes mellitus (E10-E14); gastrointestinal diseases (K00K93); neurologic diseases (G00-G99); mental and behavioral disorders (F00-F99); and musculoskeletal and connective tissue diseases (M00-M99) including osteoporosis (M80-M82).

\subsection{Statistical analysis}

The participants in the control cohort group were identified using 1:4 matching in which the nearest available person for each case was selected as a matched cohor ${ }^{14}$. We compared the baseline characteristics (age, sex, type of insurance, Charlson Comorbidity Index $)^{15}$ using the McNemar test because each asthmatic patient was matched to several non-asthmatic patients. The Kaplan-Meier method was used to estimate survival curves during the follow-up period, and survival was compared among groups using the log-rank test. Hazard ratio (HRs) with $95 \%$ confidence interval (CI) for the mortality of patients in the asthma cohort compared with subjects in the control cohort were evaluated using an age- and sex-stratified Cox regression model. To evaluate the effects of asthma-related pulmonary comorbidity (e.g., COPD, bronchiectasis, pneumonia, lung cancer, and NTM infection) on mortality in patients with asthma compared with subjects without asthma, the asthma cohort was classified into two groups based on comorbidity. Next, we performed ageand sex-adjusted Cox regression to evaluate the effects of respiratory comorbidity on HR for mortality in asthmatic patients compared with subjects without asthma. All analyses were conducted using SAS 9.4 (SAS Institute, Cary, NC, USA). All tests were two-sided, and p-values $<0.05$ were considered statistically significant.

\section{RESULTS}

\subsection{Participants}

The baseline characteristics of the asthma and control cohorts are summarized in Table 1. The mean age of patients in the asthma cohort was 49 years, and $64.1 \%$ were female. There were no significant differences regarding age and sex. Regarding the type of insurance, patients in the asthma cohort were more likely to receive medical aid compared with subjects in the control cohort. Detailed information regarding comorbidity profiles are summarized in Supplemental Table 1 .

\subsection{Mortality}

During a median 8.9-year follow-up duration, the overall mortality rate was 1,312/100,000 PY in the asthma cohort and 1,174/100,000 PY in the control cohort $(\mathrm{p}<0.001)$, which is consistent with survival analysis $(\mathrm{p}<0.01$ for a log-rank test; Figure 1 ). Patients in the asthma cohort had a significantly higher all-cause mortality rate than subjects in the control cohort $(\mathrm{HR}=1.13,95 \% \mathrm{CI}=1.07-1.19)$. As shown in Figure 2A , a positive association between asthma and all-cause mortality was observed in both age groups. However, 
the association was only significant in males, patients who received medical aid, patients with COPD, patients without bronchiectasis, and patients without pneumonia. The association was stronger in males than females ( $\mathrm{p}$ for interaction $=0.004)$, in patients who received medical aid than in patients who received other medical insurance, and in patients with COPD than in patients without COPD ( $\mathrm{p}$ for interaction $=0.003$ )

The HR for respiratory mortality was 2.63 (95\% CI = 2.28-3.03). As shown in Figure 2B , the positive association between asthma and respiratory mortality was consistent in all subgroups. The association was stronger in males than females $(\mathrm{p}$ for interaction $=0.043)$ and in younger patients (age $<60$ years) than older patients (age $\geq 60$ years; $\mathrm{p}$ for interaction $=0.005$ )

3.3. The effects of asthma-related respiratory comorbidities on mortality in the asthma cohort compared with the control cohort

As shown in Table $\mathbf{2}$, the relative risk of mortality in asthmatic patients without COPD (adjusted HR, $\mathrm{aHR}=0.95,95 \% \mathrm{CI}=0.90-1.01)$ or pneumonia $(\mathrm{aHR}=0.98,95 \% \mathrm{CI}=0.98-1.09)$ compared with the controls was not significantly increased; however, asthmatic patients with $\mathrm{COPD}(\mathrm{aHR}=1.49,95 \% \mathrm{CI}=$ $1.39-1.59)$ or pneumonia $(\mathrm{aHR}=1.59,95 \% \mathrm{CI}=1.46-1.74)$ had increased risk of mortality compared with the controls $(\mathrm{aHR}=1.49,95 \% \mathrm{CI}=1.39-1.59)$, which is in agreement with survival analyses (COPD in Figure 3A and pneumonia in Figure 3B) .

Regardless of the presence or absence of bronchiectasis or lung cancer, asthmatic patients had an increased risk of mortality compared with the controls. The presence of bronchiectasis (aHR $=1.43,95 \% \mathrm{CI}=1.18$ 1.73 ) or lung cancer ( $\mathrm{aHR}=4.11,95 \% \mathrm{CI}=2.59-6.52)$ tended to further increase the risk of all-cause mortality in the asthmatic cohort compared with the control cohort (bronchiectasis in Figure 3C and lung cancer in Figure 3D ). Although the risk of mortality was increased in asthmatic patients without NTM infection (aHR $=1.13,95 \% \mathrm{CI}=1.08-1.18$ ) compared with subjects without asthma, there was no significant increased risk of mortality in asthmatic patients without NTM infection compared with subjects without asthma ( $\mathrm{aHR}=0.85,95 \% \mathrm{CI}=0.12-6.00$; Figure 3E $)$.

Except for lung cancer, patients in the asthma cohort had a higher risk of respiratory mortality compared with subjects in the control cohort regardless of respiratory comorbidities. The presence of these respiratory comorbidities tended to further increase the risk of respiratory mortality in the asthmatic cohort compared with the control cohort (Table 2 ).

\section{DISCUSSION}

In this study, we compared the overall and respiratory mortality rates in patients with and without asthma using a large NHIS-NSC sample cohort. Patients with asthma had higher all-cause and respiratory mortality rates compared with subjects without asthma. The effects of asthma on all-cause mortality were more evident in the male subgroup, patients under medical aid, and subjects with COPD. Asthma had a more substantial effect on respiratory mortality in male subgroups and the younger population (age $<60$ years). In addition, to the best of our knowledge, this is the first study in which higher mortality in asthmatics compared with non-asthmatics was comprehensively shown to be attributed to respiratory comorbidities other than COPD (e.g., bronchiectasis, lung cancer, and pneumonia).

A previous study evaluating 164,845 asthmatic patients and matched controls showed that all-cause mortality was approximately 1.25-fold higher in asthmatic patients compared with controls ${ }^{4}$. Our study confirmed these findings with a larger number of patients from a nationally representative database, showing that asthmatic patients had 1.13-fold higher mortality compared with subjects without asthma. These findings indicate that asthma management remains unsatisfactory, and proper asthma management strategies are needed, especially in males and the younger population. The reasons for high mortality in male asthmatics might be explained by a large gap in the smoking rate between Korean males and females ${ }^{16}$. Smoking is associated with severe asthmatic symptoms and exacerbations ${ }^{17}$. Smoking is also associated with increased risk of COPD and lung cancer in asthmatic patients ${ }^{8,18}$, which subsequently can increase mortality. The reasons why the effects of asthma on respiratory mortality are higher in the younger population remain unclear. 
One hypothesis is that the effects of asthma on mortality gradually decrease as patients age. Many elderly subjects have multiple comorbidities other than asthma that can lead to mortality ${ }^{19}$. Thus, the effects of asthma on mortality in the elderly might not be as high as in the younger population.

One of the critical findings of our study is that the higher mortality in asthmatic patients compared with subjects without asthma was mainly due to comorbid respiratory diseases. Smoking-related respiratory comorbidities, such as COPD and lung cancer, were the main risk factors for increased mortality in asthmatics compared with subjects without asthma. Similarly, previous studies showed that COPD substantially increases the burden of symptoms, the rate of acute exacerbations, and the mortality rate in asthmatic patients $^{4,20-22}$. Another explanation for this phenomenon is the recent evidence linking asthma with risk of lung cancer ${ }^{8}$. Furthermore, our results showed that asthmatic patients with lung cancer had an extremely high risk of mortality compared with subjects without asthma. In addition, the risk of mortality was highest in asthmatic patients with lung cancer compared with the presence of other respiratory comorbidities. Overall, the results indicate the need for strategies to prevent smoking-related pulmonary diseases, such as smoking cessation, in management of asthmatic patients.

Bronchiectasis is a common comorbidity of asthma and is associated with severe symptoms and larger number of exacerbations ${ }^{7}$. However, the effects of bronchiectasis on mortality in asthmatic patients have rarely been reported. A recent Spanish study evaluated the effects of in-hospital bronchiectasis mortality and found that in-hospital mortality was higher in asthmatic patients with bronchiectasis than in those without bronchiectasis $(2.1 \%$ vs . $1.2 \%)$. However, the difference in mortality was not significant after adjusting for covariables ${ }^{23}$. In contrast, another study evaluated the impact of bronchiectasis on mortality in patients with severe asthma and showed that bronchiectasis was associated with all-cause mortality ${ }^{24}$. As the former study evaluated only in-hospital mortality, and the latter evaluated severe asthma patients, there are limited data on this issue. Showing the effects of bronchiectasis on increased all-cause and respiratory mortality in asthmatic patients using a nationwide large sample data is an important advantage of the present study. Notably, although coexisting NTM infection did not increase all-cause mortality in asthmatic patients compared with subjects without asthma, NTM infection was associated with increased respiratory mortality. However, because the number of patients with NTM infection was small, further studies are needed.

There are several limitations to our study. First, the NHIS-NSC database did not provide data on smoking history and pulmonary function, which are important predictors of our main outcomes. Thus, we could not adjust smoking status for the effects of these comorbidities on mortality. Second, this study was performed in Korea. Although we analyzed a large number of patients using a nationally representative sample, the data might not be applicable to other ethnic groups in different countries. However, using the nationwide representative database is also an important advantage of the study. To the best of our knowledge, this is the first study that demonstrated increased mortality and causes of mortality in asthmatics compared with subjects without asthma using nationally representative data. Third, we did not adjust for compliance with medications. However, because different inhalation doses can be administered by one inhaler according to asthma treatment protocol, it is complicated to analyze medication usage ratio. In addition, some medications (e.g., budesonide/formoterol inhaler and oral corticosteroids) are used as controllers as well as relievers.

In conclusion, the mortality in patients with asthma was higher compared with subjects without asthma, especially in patients with pulmonary comorbidities, such as COPD, bronchiectasis, lung cancer, or pneumonia. Despite the recent advancement in asthma management, strategies to improve mortality of asthma are still needed, especially in patients with pulmonary comorbidities.

\section{CONFLICTS OF INTEREST}

None to disclose

\section{AUTHOR CONTRIBUTIONS}

Y. Yeo, H. Lee, J. Ryu, and J-Y. Moon were involved in study design. Y. Yeo, H. Lee, J. Ryu, S-H Kim, 
and J-Y. Moon were involved in data analysis. Y. Yeo, H. Lee, J. Ryu, and J-Y. Moon were involved in writing the manuscript. All authors were involved in reviewing and revising the manuscript.

\section{ORCID}

Yoomi Yeo https://orcid.org/0000-0002-2447-2067

Hyun Lee https://orcid.org/0000-0002-1269-0913

Jiin Ryu https://orcid.org/0000-0001-9763-5413

Sung Jun Chung https://orcid.org/0000-0001-8636-446X

Tai Sun Park https://orcid.org/0000-0001-7383-7934

Dong Won Park https://orcid.org/0000-0002-4538-6045

Tae-Hyung Kim https://orcid.org/0000-0002-3863-7854

Jang Won Sohn https://orcid.org/0000-0001-7132-2988

Sang-Heon Kim https://orcid.org/0000-0001-8398-4444

Ho Joo Yoon https://orcid.org/0000-0002-4645-4863

Ji-Yong Moon https://orcid.org/0000-0003-2459-3448

\section{REFERENCES}

1. Ebmeier S, Thayabaran D, Braithwaite I, Benamara C, Weatherall M, Beasley R. Trends in international asthma mortality: analysis of data from the WHO Mortality Database from 46 countries (1993-2012). Lancet. 2017;390(10098):935-945.

2. Asher I, Haahtela T, Selroos O, Ellwood P, Ellwood E, Global Asthma Network Study G. Global Asthma Network survey suggests more national asthma strategies could reduce burden of asthma. Allergol Immunopathol (Madr). 2017;45(2):105-114.

3. Ellwood P, Asher MI, Billo NE, et al. The Global Asthma Network rationale and methods for Phase I global surveillance: prevalence, severity, management and risk factors. Eur Respir J. 2017;49(1).

4. Lemmetyinen RE, Karjalainen JV, But A, et al. Higher mortality of adults with asthma: A 15-year follow-up of a population-based cohort.Allergy. 2018;73(7):1479-1488.

5. Putcha N, Hansel NN. All-cause mortality in asthma. The importance of age, comorbidity, and socioeconomic status. Ann Am Thorac Soc.2014;11(8):1252-1253.

6. To T, Simatovic J, Zhu J, et al. Asthma deaths in a large provincial health system. A 10-year populationbased study. Ann Am Thorac Soc. 2014;11(8):1210-1217.

7. Padilla-Galo A, Olveira C, Fernández de Rota-Garcia L, et al. Factors associated with bronchiectasis in patients with uncontrolled asthma; the NOPES score: a study in 398 patients. Respiratory Research.2018;19(1):43.

8. Woo A, Lee SW, Koh HY, Kim MA, Han MY, Yon DK. Incidence of cancer after asthma development: 2 independent population-based cohort studies.J Allergy Clin Immunol. 2020.

9. Hojo M, Iikura M, Hirano S, Sugiyama H, Kobayashi N, Kudo K. Increased risk of nontuberculous mycobacterial infection in asthmatic patients using long-term inhaled corticosteroid therapy.Respirology. 2012;17(1):185-190.

10. Kim MH, Rhee CK, Shim JS, et al. Inhaled Corticosteroids in Asthma and the Risk of Pneumonia. Allergy Asthma Immunol Res.2019;11(6):795-805. 
11. Lee J, Lee JS, Park SH, Shin SA, Kim K. Cohort Profile: The National Health Insurance Service-National Sample Cohort (NHIS-NSC), South Korea.Int J Epidemiol. 2017;46(2):e15.

12. Cho EY, Oh KJ, Rhee CK, et al. Comparison of clinical characteristics and management of asthma by types of health care in South Korea. J Thorac Dis. 2018;10(6):3269-3276.

13. Choi JY, Yoon HK, Lee JH, et al. Current status of asthma care in South Korea: nationwide the Health Insurance Review and Assessment Service database. J Thorac Dis. 2017;9(9):3208-3214.

14. Rosenbaum PR. Observational Studies. Springer Series in Statistics, 1995.

15. Charlson ME, Pompei P, Ales KL, MacKenzie CR. A new method of classifying prognostic comorbidity in longitudinal studies: development and validation. J Chronic Dis. 1987;40(5):373-383.

16. Gunter R, Szeto E, Jeong S-H, Suh SA, Waters AJ. Cigarette Smoking in South Korea: A Narrative Review. Korean Journal of Family Medicine. 2020;41(1):3.

17. Polosa R, Thomson NC. Smoking and asthma: dangerous liaisons.European Respiratory Journal. 2013;41(3):716.

18. Aanerud M, Carsin A-E, Sunyer J, et al. Interaction between asthma and smoking increases the risk of adult airway obstruction. European Respiratory Journal. 2015;45(3):635-643.

19. Camilloni L, Farchi S, Giorgi Rossi P, Chini F, Borgia P. Mortality in elderly injured patients: the role of comorbidities.International journal of injury control and safety promotion.2008;15(1):25-31.

20. Lee H, Kim S-H, Kim B-K, et al. Characteristics of Specialist-Diagnosed Asthma-COPD Overlap in Severe Asthma: Observations from the Korean Severe Asthma Registry (KoSAR). Allergy.2020;n/a(n/a).

21. Lee H, Ryu J, Chung SJ, et al. Coexisting COPD Increases Mortality in Patients With CorticosteroidDependent Asthma: A Nationwide Population-Based Study. Allergy Asthma Immunol Res.2020;12(5):821831.

22. Leung JM, Sin DD. Asthma-COPD overlap syndrome: pathogenesis, clinical features, and therapeutic targets. Bmj. 2017;358:j3772.

23. Sánchez-Muñoz G, López-de-Andrés A, Jiménez-García R, et al. Trend from 2001 to 2015 in the prevalence of bronchiectasis among patients hospitalized for asthma and effect of bronchiectasis on the in-hospital mortality. J Asthma. 2020:1-10.

24. Cho H, Lee H, Ryu J, Chung SJ, Park DW, Sohn JW, Yoon HJ, Kim SH. Bronchiectasis and Increased Mortality in Patients with Corticosteroid-Dependent Severe Asthma: A Nationwide Population Study. Therapeutic Advances in Respiratory Disease 2020 [in submission].

TABLE 1. Baseline descriptive characteristics of the study population

\begin{tabular}{lllll}
\hline Total $(\mathrm{n}=96,595)$ & $\begin{array}{l}\text { Asthma cohort } \\
=19,319)\end{array}$ & $\begin{array}{l}\text { Control cohort }(\mathrm{n}= \\
77,276)\end{array}$ & $\mathrm{p}$-value \\
$\begin{array}{l}\text { Age, years } \\
\text { Age group }\end{array}$ & $49(36-64)$ & $49(36-64)$ & \\
$\begin{array}{l}\text { Twenties } \\
\text { Thirties }\end{array}$ & $11,880(12.3)$ & $2,376(12.3)$ & $9,504(12.3)$ & \\
Forties & $19,255(19.9)$ & $3,851(19.9)$ & $15,404(19.9)$ & \\
Fifties & $18,370(19.0)$ & $3,674(19.0)$ & $14,696(19.0)$ & \\
Sixties & $15,750(16.3)$ & $3,150(16.3)$ & $12,600(16.3)$ & \\
Seventies & $16,610(17.2)$ & $3,322(17.2)$ & $13,288(17.2)$ & \\
Sex & $14,730(15.3)$ & $2,946(15.3)$ & $11,784(15.3)$ & \\
Male & $34,720(35.9)$ & $6,944(35.9)$ & $27,776(35.9)$ & \\
& & &
\end{tabular}




\begin{tabular}{lccc}
$\begin{array}{l}\text { Female } \\
\text { Type of } \\
\text { insurance }\end{array}$ & $61,875(64.1)$ & $12,375(64.1)$ & $49,500(64.1)$ \\
$\begin{array}{l}\text { Self-employed } \\
\text { health insurance }\end{array}$ & $39,519(40.9)$ & $7,369(38.1)$ & $32,150(41.6)$ \\
$\begin{array}{l}\text { Employee health } \\
\text { insurance }\end{array}$ & $52,047(53.9)$ & $10,198(52.8)$ & $41,849(54.2)$ \\
Medical aid & $5,029(5.2)$ & $1,752(9.1)$ & $3,277(4.2)$ \\
\hline
\end{tabular}

Data are presented as frequency (\%) and mean with standard deviation.

COPD, chronic obstructive pulmonary disease

*Asthma-related medication included inhaled corticosteroid (32\%), long-acting $\beta 2$ agonist (19.1\%), shortacting $\beta 2$ agonist (34.8\%), and leukotriene receptor antagonist (35.7\%).

TABLE 2. The effects of asthma and its coexisting respiratory comorbidities on mortality in patients with asthma compared with subjects without asthma

\begin{tabular}{lll}
\hline & $\begin{array}{l}\text { Mortality } \\
\text { All-cause }\end{array}$ & $\begin{array}{l}\text { Mortality } \\
\text { Respiratory disease-related }\end{array}$ \\
& HR $(95 \%$ CI $)$ & HR $(95 \%$ CI $)$ \\
COPD & Ref & Ref \\
Control cohort & $0.95(0.90-1.01)$ & $1.58(1.32-1.89)$ \\
Asthma cohort without COPD & $1.49(1.39-1.59)$ & $4.27(3.66-4.99)$ \\
Asthma cohort with COPD & & \\
$\begin{array}{l}\text { Bronchiectasis } \\
\text { Control cohort }\end{array}$ & Ref & Ref \\
Asthma cohort without bronchiectasis & $1.12(1.06-1.17)$ & $2.38(2.08-2.72)$ \\
Asthma cohort with bronchiectasis & $1.43(1.18-1.73)$ & $6.35(4.59-8.78)$ \\
Lung cancer & & \\
Control cohort & Ref & Ref \\
Asthma cohort without lung cancer & $1.12(1.07-1.18)$ & $2.55(2.24-2.90)$ \\
Asthma cohort with lung cancer & $4.11(2.59-6.52)$ & $13.25(0.46-23.09)$ \\
Pneumonia & & \\
Control cohort & Ref & Ref \\
Asthma cohort without pneumonia & $1.03(0.98-1.09)$ & $2.08(1.79-2.41)$ \\
Asthma cohort with pneumonia & $1.59(1.46-1.74)$ & $4.72(3.89-5.73)$ \\
NTM infection & & \\
Control cohort & Ref & Ref \\
Asthma cohort without NTM infection & $1.13(1.08-1.18)$ & $2.54(2.23-2.89)$ \\
Asthma cohort with NTM infection & $0.85(0.12-6.00)$ & $8.45(1.19-60.21)$ \\
\hline
\end{tabular}

Data are presented as a ratio $(95 \% \mathrm{CI})$.

$\mathrm{HR}$, hazard ratio; CI, confidence interval; COPD, chronic obstructive pulmonary disease; NTM, nontuberculous mycobacteria

FIGURE LEGENDS

FIGURE 1. Kaplan-Meier survival plot of time to death in the asthma cohort versus the control cohort.

FIGURE 2. Subgroup analysis for the effects of asthma on mortality. A) all-cause mortality B) Respiratory 
mortality

FIGURE 3. Kaplan-Meier survival plot of time to death in asthmatic patients with comorbidities, asthmatic patients without comorbidities, and subjects without asthma. A) COPD, B) pneumonia, C) bronchiectasis, D) lung cancer, E) NTM infection

COPD, chronic obstructive pulmonary disease; NTM, non-tuberculous mycobacter

Supplemental materials

Supplemental TABLE 1. Baseline comorbidity profiles of the study population

Supplemental Figure 1. Flow chart

\section{Hosted file}

Figure_Final.pdf available at https://authorea.com/users/370233/articles/488924-coexistingrespiratory-comorbidities-increase-mortality-in-patients-with-asthma-a-national-cohortstudy 